\title{
Prostaglandin E2-EP2-NF-kB signaling in macrophages mediates chronic inflammation during intracranial aneurysm formation and becomes a potential therapeutic target
}

\author{
Tomohiro Aoki ${ }^{1,2,3}$, Juhana Frosen ${ }^{4,5}$, Shuh Narumiya ${ }^{2,3}$
}

\begin{abstract}
${ }^{\prime}$ Department of Molecular Pharmacology, Research Institute, National Cerebral and Cardiovascular Center, Japan, ${ }^{2}$ Alliance Laboratory for Advanced Medical Research, Medical Innovation Center, Kyoto University Graduate School of Medicine, Japan, ${ }^{3}$ Core Research for Evolutional Science and Technology (CREST), Medical Innovation Center, Kyoto University Graduate School of Medicine, Japan, ${ }^{4}$ Neurosurgery Research Group, Biomedicum Helsinki, Finland, ${ }^{5}$ NeuroCenter, Kuopio University Hospital, Finland
\end{abstract}

Intracranial aneurysm (IA) is a common disease affecting 1 to $5 \%$ among the general public and causes subarachnoid hemorrhage after rupture as a major cause. Given a poor prognosis of subarachnoid hemorrhage once after the onset, prevention of IA progression is critical for social health. Analysis of surgically dissected human IA specimens and studies in animal models suggest the crucial contribution of macrophage-mediated chronic inflammation to the pathogenesis of IAs. Animal studies further implicate NF-kB activation and prostaglandin E2 (PGE2)-prostaglandin E receptor subtype 2 (EP2) signaling in this process. However, detailed machineries how PGE2-EP2 signaling or NF-kB activation functions to drive the pathogenesis of IAs remain to be elucidated. Here, we demonstrated that both EP2 and cyclooxygenase-2 (COX -2), an enzyme producing PGE2, expression were detected in human IA lesions and the presence of these two proteins was positively correlated with macrophage infiltration in situ. Next, we monitored the spatiotemporal pattern of NF-kB activation during IA development in NF-kB-reporter mice and showed that NF-kB was first activated in endothelial cells and in macrophages infiltrating in the adventitia and that, later on, activation spread to the entire arterial wall. Specific deletion of Ptger2 (which encodes EP2) in macrophages, not in endothelial cells, prevented macrophage infiltration and NF-kB activation in the lesion and suppressed IA formation. Induction of an IkBa mutant specifically in macrophages suppressed IA formation and macrophage infiltration as well. In cultured cells, EP2 signaling cooperated with TNF-a to synergistically induce inflammation-related genes such as COX-2 through forming the transcription factor complex including NF-kB. Induction of COX-2 resulted in the formation of positive feedback loop including EP2 signaling, making PGE2-dependent inflammation being amplified and chronic. Furthermore, EP2 signaling stabilized MCP-1 mRNA via activating RNA-binding protein HuR. This signaling in macrophages thus may contribute to the formation of autoamplification loop among macrophages. Finally, administration of an EP2 antagonist, PF-04418948, in rats suppressed macrophage infiltration and prevented IA formation and progression. PGE2-EP2-NF-\&\#61547;B-COX-2 signaling in macrophages thus facilitates IA development by mediating the chronicity of inflammation and providing the inflammatory microenvironment in intracranial arteries. EP2 may therefore be a therapeutic target for IAs. 